\title{
Biosynthesis of metal nanoparticles using blue-green algae (Cyanobacteria) and their possible applications
}

\author{
Rehab S. Ghobashy, Mostafa M. Elsheekh, Gehan A. Ismail and Saly F. Gheda
}

Botany Department, Faculty of Science, Tanta University, Tanta, Egypt

\section{III}

Background: Silver nanoparticles has attracted much attention as its appropriate solvent systems and ecological reducing agents so, its synthesis was of wide prominence and were functional in many fields. Aim: The present work was designed for the biosynthesis of AgNPs by seven cyanobacterial species using their filtrate and biomass separately and also their crude phycobiliproteins extract of Nostoc linckia and Spirulina platensis which scored the highest quantities. Results: Appearance of surface plasmon band at $400 \mathrm{~nm}$ for filtrate and biomass while at $420 \mathrm{~nm}$ crude phycobiliproteins extract that indicated the biosynthesis of AgNPs. FTIR analysis suggested that proteins were responsible for their capping and stabilization. XRD analysis confirmed its spherical crystalline shape. Zeta potential recorded $-15.9 \mathrm{mV}$ and $-16.8 \mathrm{mV}$ for S. platensis and $N$. linckia AgNPs, respectively confirming its stability. TEM images revealed that AgNPs had a mean average size of 21.2 and 21.05 $\mathrm{nm}$ for S. platensis and N. linckia, respectively. AgNPs showed potent antimicrobial activity against Gram positive, Gram negative bacteria and Candida albicans using the disk diffusion method. The antioxidant activity of AgNPs was promising by using three assays of DPPH radical scavenging activity, total antioxidant capacity and ferric reducing antioxidant power. The cytotoxicity of AgNPs was tested on HepG2 cell line through neutral red assay and the NPs of both species proved to be safe in low concentrations. Significant antiviral activity against HCV (64.976\%) was recorded for AgNPs of N. linckia which was comparable to Ribavirin (66.67\%) as a standard drug while S. platensis AgNPs recorded 48.3\%. Conclusion: Biosynthesis of metal nanoparticles using blue-green algae (Cyanobacteria) can express potential anti-cancer activities.

Keywords: Antiviral; AgNPs; Antimicrobial; Antioxidant; Cyanobacteria

Editor-in-Chief: Prof. M.L. Salem, PhD - Article DOI: 10.21608/JCBR.2021.59664.1133 